TRANSACTIONS OF THE

AMERICAN MATHEMATICAL SOCIETY

Volume 363, Number 4, April 2011, Pages 1743-1763

S 0002-9947(2010)05238-9

Article electronically published on November 5, 2010

\title{
THE ENERGY OF EQUIVARIANT MAPS AND A FIXED-POINT PROPERTY FOR BUSEMANN NONPOSITIVE CURVATURE SPACES
}

\author{
MAMORU TANAKA
}

\begin{abstract}
For an isometric action of a finitely generated group on the ultralimit of a sequence of global Busemann nonpositive curvature spaces, we state a sufficient condition for the existence of a fixed point of the action in terms of the energy of equivariant maps from the group into the space. Furthermore, we show that this energy condition holds for every isometric action of a finitely generated group on any global Busemann nonpositive curvature space in a family which is stable under ultralimit, whenever each of these actions has a fixed point.

We also discuss the existence of a fixed point of affine isometric actions of a finitely generated group on a uniformly convex, uniformly smooth Banach space in terms of the energy of equivariant maps.
\end{abstract}

\section{INTRODUCTION}

One of the purposes of this paper is to generalize results in 6] and 7] for Hadamard spaces to global Busemann nonpositive curvature spaces: For a family of global Busemann nonpositive curvature spaces which is stable under ultralimit, we investigate whether any isometric action of a finitely generated group on any space in the family has a fixed point, in terms of the energy of equivariant maps from the group into spaces in the family.

Let $\Gamma$ be a finitely generated group and $\rho$ a homomorphism from $\Gamma$ into the full isometry group of a global Busemann nonpositive curvature space (Definition 4.1). The homomorphism $\rho$ can be regarded as an isometric action on the space. For the homomorphism $\rho$, we define a generalized nonnegative energy functional $E$ (Definition 3.1) on the space of the $\rho$-equivariant maps from $\Gamma$ into the space. The energy functional vanishes at a $\rho$-equivariant map if and only if the image of the map is a fixed point of $\rho(\Gamma)$.

In [7, the gradient flow of the energy of equivariant maps into Hadamard spaces is used to investigate the existence of a fixed point. The gradient flow was introduced by Jost in 9 and Mayer in 11. It generates a strongly continuous semigroup of Lipschitz-continuous mappings and decreases the energy in the most efficient way.

Received by the editors May 16, 2008.

2010 Mathematics Subject Classification. Primary 58E20, 58E40; Secondary 51F99.

Key words and phrases. Global Busemann NPC spaces, fixed point, ultralimits, uniformly convex, uniformly smooth Banach space.

The author was supported by Grant-in-Aid for JSPS Fellows (21·1062).

(C)2010 American Mathematical Society Reverts to public domain 28 years from publication 
Although it seems that it is difficult to generalize Jost-Mayer's gradient flow to global Busemann nonpositive curvature spaces, we can consider the absolute gradient $\left|\nabla_{-} E\right|(f)$ of the energy functional $E$ at each $\rho$-equivariant map $f$ (Definition 5.1). The absolute gradient gives the maximum descent of the energy functional around each map with respect to a natural distance on the space of $\rho$-equivariant maps. The absolute gradient $\left|\nabla_{-} E\right|(f)$ can be regarded as the norm of the gradient of $E$ at $f$. If the absolute gradient vanishes at a $\rho$-equivariant map, then the map minimizes the energy functional. We can always find a sequence of $\rho$-equivariant maps such that the absolute gradient approaches 0 (Lemma 5.4).

For a nonprincipal ultrafilter on $\mathbb{N}$ (Definition 2.1), the ultralimit of a sequence of global Busemann nonpositive curvature spaces with base points is always defined (Definition 2.2). The ultralimit is also defined for a sequence of homomorphisms from $\Gamma$ into the full isometry groups of the spaces, equivariant maps, and energies under a certain condition.

Theorem 1. Let $\rho$ be a homomorphism from $\Gamma$ into the full isometry group of a global Busemann nonpositive curvature space $(N, d)$. Denote by $E$ the energy functional on the space of $\rho$-equivariant maps. Take a sequence of $\rho$-equivariant maps $f_{n}$ such that the absolute gradient of $E$ approaches 0 . If there exists a positive constant $C$ such that

$$
\left|\nabla_{-} E\right|\left(f_{n}\right)^{2} \geq C E\left(f_{n}\right)
$$

holds for every $n$, then, for any nonprincipal ultrafilter on $\mathbb{N}$, there exists a fixed point of the ultralimit of the sequence of homomorphisms $\rho_{n} \equiv \rho$ in the ultralimit of the sequence of spaces $\left(N_{n}, d_{n}\right) \equiv(N, d)$ with base points $f_{n}(e)$. Here e denotes the identity element of $\Gamma$.

In the case of a Hadamard space, in [7, they prove that if the assumptions in Theorem 1 hold, then there exists a fixed point of the image of the original homomorphism $\rho$ in the original space. Let $\mathcal{L}$ be a family of global Busemann nonpositive curvature spaces which is stable under ultralimit (Definition 2.3).

Theorem 2. Suppose that, for any $(N, d) \in \mathcal{L}$ and homomorphism $\rho$ from $\Gamma$ into the full isometry group of $(N, d)$, there exists a fixed point of $\rho(\Gamma)$. Then there exists a positive constant $C$ such that

$$
\left|\nabla_{-} E_{\rho}\right|(f)^{2} \geq C E_{\rho}(f)
$$

holds for all $(N, d) \in \mathcal{L}$, homomorphisms $\rho$ from $\Gamma$ into the full isometry group of $(N, d)$, and $\rho$-equivariant maps $f$ from $\Gamma$ into $N$. Here $E_{\rho}$ denotes the energy functional for $\rho$-equivariant maps. The constant $C=C(\Gamma, \mathcal{L})$ should be independent of $(N, d), \rho$, and $f$.

Examples of a family of global Busemann nonpositive curvature spaces which is stable under ultralimit are given in Section 6. In [6, they prove Theorem 2 in the case of a family of Hadamard spaces which is stable under ultralimit. It is well known that if $\mathcal{L}$ is the family of all Hilbert spaces, then the so-called Kazhdan's property $(T)$ is equivalent to the existence of a constant in Theorem 2. Hence the maximum of the constant $C(\Gamma, \mathcal{L})$ in Theorem 2 can be regarded as a generalization of the Kazhdan constant of $\Gamma$.

Another purpose of this paper is to consider the existence of a fixed point of affine isometric actions of a finitely generated group $\Gamma$ on a Banach space $(B,\|\|)$ in terms 
of the energy of equivariant maps. Let $\pi$ be a linear isometric $\Gamma$-representation on $B$, that is, a homomorphism from $\Gamma$ into the full linear isometry group of $(B,\|\|)$. Denote by $B^{\pi(\Gamma)}$ the closed subspace of fixed vectors of $\pi$. The $\Gamma$ representation $\pi$ descends to a linear isometric $\Gamma$-representation $\pi^{\prime}$ on the quotient space $B / B^{\pi(\Gamma)}$. Let $\rho$ be an affine isometric $\Gamma$-representation on $B$, that is, $\rho$ is written as $\rho(\gamma) v=\rho_{0}(\gamma) v+o(\gamma)$ by a linear isometric $\Gamma$-representation $\rho_{0}$ on $B$ and a map $o$ from $\Gamma$ into B. Note that an isometry on a real Banach space is affine; see 3. The $\Gamma$-representation $\rho$ also descends to an affine isometric $\Gamma$-representation $\rho^{\prime}$ on $B / B^{\rho_{0}(\Gamma)}$. Denote by $E_{\rho^{\prime}}$ the energy functional on the space of all $\rho^{\prime}$-equivariant maps.

Definition ([1]). (i) We say that $\Gamma$ has property $\left(F_{B}\right)$ if any affine isometric $\Gamma$ representation on $B$ has a fixed point.

(ii) We say that $\Gamma$ has property $\left(T_{B}\right)$ if, for any nontrivial linear isometric $\Gamma$ representation $\pi$ on $B$, there exists a positive constant $C(\pi)$ such that

$$
\max _{\gamma \in S}\left\|v-\pi^{\prime}(\gamma) v\right\| \geq C(\pi)
$$

for all $v \in B / B^{\pi(\Gamma)}$ satisfying $\|v\|=1$, where $S$ is a finite generating subset of $\Gamma$.

In [1, they pointed out that, according to a theorem by Guichardet, if $\Gamma$ has property $\left(F_{B}\right)$, then it has property $\left(T_{B}\right)$. In this paper, we introduce a new property defined in terms of the energy of equivariant maps and investigate a relation between property $\left(F_{B}\right)$ and the new one.

Definition. We say that $\Gamma$ has property $\left(E_{B}\right)$ if there exists a positive constant $C(\rho)$ for any affine isometric $\Gamma$-representation $\rho$ on $B$ such that

$$
\left|\nabla_{-} E_{\rho^{\prime}}\right|(f)^{2} \geq C(\rho) E_{\rho^{\prime}}(f)
$$

for all $\rho^{\prime}$-equivariant maps $f$.

Theorem 3. If $\Gamma$ has property $\left(F_{B}\right)$, then it has property $\left(E_{B}\right)$.

There exists a finitely generated Abelian group which has property $\left(T_{B}\right)$ but does not have property $\left(F_{B}\right)$ for some Banach space $B$; see [1, Example 2.22]. However, as is well known, a finitely generated group has property $\left(T_{H}\right)$ for all Hilbert spaces $H$ (i.e., has Kazhdan's property $(T))$ if and only if it has property $\left(F_{H}\right)$ for all Hilbert spaces $H$. Furthermore, these are also equivalent to having property $\left(E_{H}\right)$ for all Hilbert spaces $H$. Thus one may expect that there is some relation between property $\left(T_{B}\right)$ and property $\left(E_{B}\right)$ for a Banach space $B$. But there is a finitely generated group which has property $\left(T_{B}\right)$ but does not have property $\left(E_{B}\right)$ for some Banach space $B$, and the author does not know whether a finitely generated group which has property $\left(E_{B}\right)$ has property $\left(T_{B}\right)$. However we can show Proposition 4 below, which gives a relation between property $\left(T_{B}\right)$ and a property which is somewhat weaker than property $\left(E_{B}\right)$.

Definition. We say that $\Gamma$ has property $\left(E_{B}\right)^{\prime}$ if, for any affine isometric $\Gamma$ representation $\rho$ on $B$ whose linear part $\rho_{0}$ is nontrivial, there exists a positive constant $C(\rho)$ such that

$$
\left|\nabla_{-} E_{\rho^{\prime}}\right|(f)^{2} \geq C(\rho) E_{\rho^{\prime}}(f)
$$

for all $\rho^{\prime}$-equivariant maps $f$. 
Proposition 4. Let $\Gamma$ be a finitely generated Abelian group, and $B$ a uniformly convex, uniformly smooth Banach space. If $\Gamma$ has property $\left(T_{B}\right)$, then it has property $\left(E_{B}\right)^{\prime}$.

From Proposition 4, we can show that there exists a finitely generated Abelian group which has property $\left(E_{B}\right)^{\prime}$ but does not have property $\left(F_{B}\right)$ for some Banach space $B$.

The paper is organized as follows: We review the definitions and properties of the ultralimit of a sequence of metric spaces in Section 2 the energy of equivariant maps in Section 3, global Busemann nonpositive curvature spaces in Section 4 and the absolute gradient in Section 5 . We show Theorem 1 and Theorem 2 in Section 6. In Section 7 we review the definitions of the properties $\left(F_{B}\right),\left(T_{B}\right),\left(E_{B}\right)$ and $\left(E_{B}\right)^{\prime}$, and show Theorem 3 and Proposition 4 .

The author would like to express his gratitude to Professor Hiroyasu Izeki for suggesting this topic and helpful comments.

\section{Ultralimit of Metric SPACES}

In this section, we recall the definition and some properties of the ultralimit of a sequence of metric spaces. Basic references of this section are [2], [10, and 6].

Definition 2.1. A nonprincipal ultrafilter $\omega$ on $\mathbb{N}$ is a subset of $2^{\mathbb{N}}$ satisfying the following: (i) $\emptyset \notin \omega$ and $\mathbb{N} \in \omega$; (ii) if $A \in \omega$ and $A \subset B$, then $B \in \omega$; (iii) if $A \in \omega$ and $B \in \omega$, then $A \cap B \in \omega$; (iv) $A \in \omega$ or $\mathbb{N} \backslash A \in \omega$ for any subset $A \subset \mathbb{N}$; (v) $B \notin \omega$ for any finite subset $B \subset \mathbb{N}$.

An example of a nonprincipal ultrafilter on $\mathbb{N}$ can be given as follows: The set $\omega^{\prime}:=\{B \subset \mathbb{N}:|\mathbb{N} \backslash B|<\infty\}$ satisfies (i), (ii), (iii), and (v). Considering all $\omega \subset 2^{\mathbb{N}}$ satisfying (i), (ii), (iii), (v), and $\omega^{\prime} \subset \omega$, we obtain a maximal one, $\omega_{0}$, by Zorn's Lemma. If there exists $A \subset \mathbb{N}$ such that $A \notin \omega_{0}$ and $\mathbb{N} \backslash A \notin \omega_{0}$, then the set $\omega_{0} \cup\left\{C \subset \mathbb{N}: A \cap B \subset C\right.$ for some $\left.B \in \omega_{0}\right\}$ also satisfies (i), (ii), (iii), and (v). The set $\omega_{0}$ is contained properly in this set. This contradicts that $\omega_{0}$ is maximal. Hence $\omega_{0}$ is a nonprincipal ultrafilter.

Let $\omega$ be a nonprincipal ultrafilter on $\mathbb{N}$. For any bounded sequence of real numbers $a_{n}$ there exists a unique real number $l$ such that $\left\{n:\left|a_{n}-l\right|<\epsilon\right\} \in \omega$ for any $\epsilon>0$. Denote $l$ by $a_{\omega}$ or $\omega-\lim _{n} a_{n}$. If a sequence $a_{n}$ converges to a real number $a$ in the usual sense, then $a_{\omega}=a$. Thus we can regard $a_{\omega}$ as a limit of $a_{n}$ in some sense. Moreover, $\omega$-lim is linear, and if a pair of bounded sequences $a_{n}, b_{n}$ satisfies $a_{n} \leq b_{n}$ for every $n$, then $a_{\omega} \leq b_{\omega}$ holds.

Definition 2.2. Let $\omega$ be a nonprincipal ultrafilter on $\mathbb{N}$ and $\left(M_{n}, d_{n}\right)$ a sequence of metric spaces with base points $o_{n} \in M_{n}$. Denote by $M_{\infty}$ the set of sequences $\left(p_{n}\right)$ such that $p_{n} \in M_{n}$ and $d_{n}\left(p_{n}, o_{n}\right)$ is bounded independently of $n$. We say that $\left(p_{n}\right)$ and $\left(q_{n}\right) \in M_{\infty}$ are equivalent if $\omega-\lim _{n} d_{n}\left(p_{n}, q_{n}\right)=0$. Let $p_{\omega}$ or $\omega-\lim _{n} p_{n}$ denote the equivalence class of $\left(p_{n}\right)$, and $M_{\omega}$ denote the set of the equivalence classes. Endow $M_{\omega}$ with a metric $d_{\omega}\left(p_{\omega}, q_{\omega}\right):=\omega-\lim _{n} d_{n}\left(p_{n}, q_{n}\right)$. We also write $\left(M_{\omega}, d_{\omega}\right)=\omega-\lim _{n}\left(M_{n}, d_{n}, o_{n}\right)$, and call it the ultralimit of a sequence of metric spaces $\left(M_{n}, d_{n}\right)$ with base points $o_{n}$ with respect to $\omega$.

The ultralimit of a sequence of metric spaces is complete. Note that if a Banach space $(B,\|\|)$ is finite dimensional, then $\omega-\lim _{n}(B,\|\|, o)$ is isometric to $(B,\|\|)$. But if $(B,\|\|)$ is infinite dimensional, then $\omega-\lim _{n}(B,\|\|, o)$ may not be isometric to $(B,\|\|)$. 
Definition 2.3. A family $\mathcal{L}$ of metric spaces is said to be stable under ultralimit if for any sequence of metric spaces $\left(N_{n}, d_{n}\right)$ in $\mathcal{L}$ with base points $o_{n}$ and any sequence of positive real numbers $r_{n}$, there exists a nonprincipal ultrafilter $\omega$ on $\mathbb{N}$ such that $\omega-\lim _{n}\left(N_{n}, r_{n} d_{n}, o_{n}\right)$ is in $\mathcal{L}$.

\section{ENERGY FUNCTIONALS AND HARMONIC MAPS}

In this section, we define the space of equivariant maps from a finitely generated group into a metric space, the energy functional on the space. A basic reference of this section is 6 .

Let $\Gamma$ be a finitely generated group, $S$ the finite generating subset of $\Gamma$ which satisfies $S=S^{-1}:=\left\{s^{-1}: s \in S\right\}$ and does not contain the identity element $e$ of $\Gamma$. Let $m: S \rightarrow(0,1)$ be a function satisfying $\sum_{\gamma \in S} m(\gamma)=1$ and $m(\gamma)=m\left(\gamma^{-1}\right)$ for every $\gamma \in S$. The function $m$ is called a weight on $S$. An isometry on a metric space $(M, d)$ is a map $g: M \rightarrow M$ which is surjective and satisfies $d(g(x), g(y))=d(x, y)$ for all $x, y \in M$. Denote by $\operatorname{Isom}(M, d)$ the group which consists of all isometries on $M$. For a homomorphism $\rho: \Gamma \rightarrow \operatorname{Isom}(M, d), \mathcal{M}$ denotes the space of $\rho$-equivariant maps from $\Gamma$ into $M$, where $\rho$-equivariant means that $f(\gamma \alpha)=\rho(\gamma) f(\alpha)$ for every $\gamma, \alpha \in \Gamma$. Endow $\mathcal{M}$ with a metric $d_{\mathcal{M}}(f, g)=d_{\mathcal{M}}(f(e), g(e))$. Then we can identify $\left(\mathcal{M}, d_{\mathcal{M}}\right)$ with $(M, d)$ through the correspondence $f \mapsto f(e)$.

Definition 3.1. We define an energy functional $E: \mathcal{M} \rightarrow[0, \infty)$ by

$$
E(f):=\frac{1}{2} \sum_{\gamma \in S} m(\gamma) d(f(e), f(\gamma))^{2}
$$

for $f \in \mathcal{M}$.

The energy functional is continuous on $\left(\mathcal{M}, d_{\mathcal{M}}\right)$. A $\rho$-equivariant map $f$ is called harmonic if $f$ minimizes $E$. Note that $E(f)=0$ if and only if $f(e)$ is a fixed point of $\rho(\Gamma)$.

Let $\omega$ be a nonprincipal ultrafilter on $\mathbb{N}$. Let $\left(M_{n}, d_{n}\right)$ be a sequence of metric spaces with base points $o_{n}$, and $\rho_{n}: \Gamma \rightarrow \operatorname{Isom}\left(M_{n}, d_{n}\right)$ a sequence of homomorphisms. It is easy to see that the following Lemma 3.2 and Lemma 3.3 hold.

Lemma 3.2. Suppose that, for every $\gamma \in \Gamma, d_{n}\left(o_{n}, \rho_{n}(\gamma) o_{n}\right)$ is bounded independently of $n$. Then we can define a homomorphism $\rho_{\omega}: \Gamma \rightarrow \operatorname{Isom}\left(M_{\omega}, d_{\omega}\right)$ by $\rho_{\omega}(\gamma) p_{\omega}:=\omega-\lim _{n}\left(\rho_{n}(\gamma) p_{n}\right)$ for every $\gamma \in \Gamma$ and $p_{\omega} \in M_{\omega}$, where $\left(p_{n}\right) \in M_{\infty}$ is a representative of $p_{\omega}$.

Denote by $\mathcal{M}_{n}$ the set of all $\rho_{n}$-equivariant maps from $\Gamma$ into $M_{n}$, and by $\mathcal{M}_{\omega}$ the set of all $\rho_{\omega}$-equivariant maps from $\Gamma$ into $M_{\omega}$. Denote by $\mathcal{M}_{\infty}$ the set of all sequences $f_{n} \in \mathcal{M}_{n}$ such that $d_{n}\left(o_{n}, f_{n}(e)\right)$ is bounded independently of $n$.

Lemma 3.3. Suppose that, for every $\gamma \in \Gamma, d_{n}\left(o_{n}, \rho_{n}(\gamma) o_{n}\right)$ is bounded independently of $n$. For $\left(f_{n}\right) \in \mathcal{M}_{\infty}$, the limit map $f_{\omega}: \Gamma \rightarrow M_{\omega}$ defined by $f_{\omega}(\gamma):=\omega$ $\lim _{n} f_{n}(\gamma)$ is $\rho_{\omega}$-equivariant. Conversely, for any $f \in \mathcal{M}_{\omega}$, there exists a representative $\left(f_{n}\right) \in \mathcal{M}_{\infty}$ of $f$.

Denote by $E_{n}$ the energy functional on $\mathcal{M}_{n}$.

Lemma 3.4. Suppose that $f_{\omega} \in \mathcal{M}_{\omega}$ has a representative $\left(f_{n}\right) \in \mathcal{M}_{\infty}$ for which $E_{n}\left(f_{n}\right)$ is bounded independently of $n$. Then the energy functional $E_{\omega}: \mathcal{M}_{\omega} \rightarrow$ 
$[0, \infty)$ defined by

$$
E_{\omega}\left(f_{\omega}\right)=\frac{1}{2} \sum_{\gamma \in S} m(\gamma) d_{\omega}\left(f_{\omega}(e), f_{\omega}(\gamma)\right)^{2}
$$

satisfies $E_{\omega}\left(f_{\omega}\right)=\omega-\lim _{n} E_{n}\left(f_{n}^{\prime}\right)$ for any representative $\left(f_{n}^{\prime}\right) \in \mathcal{M}_{\infty}$ of $f_{\omega}$.

Proof. By assumption, $d_{n}\left(f_{n}(e), f_{n}(\gamma)\right)$ is bounded independently of $n$ and $\gamma \in S$. Hence there exists $C^{\prime}>0$ such that $d_{n}\left(f_{n}(e), f_{n}(\gamma)\right)<C^{\prime}$ for every $n$ and $\gamma \in S$. Thus $d_{n}\left(f_{n}(e), f_{n}(\gamma)\right)+d_{\omega}\left(f_{\omega}(e), f_{\omega}(\gamma)\right) \leq 2 C^{\prime}$ holds. For any $\epsilon>0$, we have

$$
\begin{aligned}
& \left\{n \in \mathbb{N}:\left|\sum_{\gamma \in S} m(\gamma) d_{n}\left(f_{n}(e), f_{n}(\gamma)\right)^{2}-\sum_{\gamma \in S} m(\gamma) d_{\omega}\left(f_{\omega}(e), f_{\omega}(\gamma)\right)^{2}\right|<\epsilon\right\} \\
\supset & \left\{n \in \mathbb{N}: \sum_{\gamma \in S} m(\gamma)\left|d_{n}\left(f_{n}(e), f_{n}(\gamma)\right)^{2}-d_{\omega}\left(f_{\omega}(e), f_{\omega}(\gamma)\right)^{2}\right|<\epsilon\right\} \\
\supset & \bigcap_{\gamma \in S}\left\{n \in \mathbb{N}: m(\gamma)\left|d_{n}\left(f_{n}(e), f_{n}(\gamma)\right)^{2}-d_{\omega}\left(f_{\omega}(e), f_{\omega}(\gamma)\right)^{2}\right|<\frac{\epsilon}{|S|}\right\} \\
\supset & \bigcap_{\gamma \in S}\left\{n \in \mathbb{N}:\left|d_{n}\left(f_{n}(e), f_{n}(\gamma)\right)-d_{\omega}\left(f_{\omega}(e), f_{\omega}(\gamma)\right)\right|<\frac{\epsilon}{2 C^{\prime} m(\gamma)|S|}\right\},
\end{aligned}
$$

where $|S|$ is the number of the elements of $S$. Since the set at the last line is in $\omega$, we get

$$
\left\{n \in \mathbb{N}:\left|E_{n}\left(f_{n}\right)-\frac{1}{2} \sum_{\gamma \in S} m(\gamma) d_{\omega}\left(f_{\omega}(e), f_{\omega}(\gamma)\right)^{2}\right|<\epsilon\right\} \in \omega
$$

for any $\epsilon>0$. Take a representative $\left(f_{n}^{\prime}\right) \in \mathcal{M}_{\infty}$ of $f_{\omega}$. Put $K_{\epsilon}:=\{n \in \mathbb{N}$ : $\left.d_{n}\left(f_{n}, f_{n}^{\prime}\right)<\epsilon\right\}$ for $\epsilon>0$. Using the triangle inequality and the Hölder inequality, we have

$$
E_{n}(f)^{1 / 2} \leq E_{n}(g)^{1 / 2}+\sqrt{2} d_{\mathcal{M}_{n}}(f, g)
$$

for all $f, g \in \mathcal{M}_{n}$. Hence we obtain

$$
\begin{aligned}
\left|E_{n}\left(f_{n}\right)-E_{n}\left(f_{n}^{\prime}\right)\right| & =\left|E_{n}\left(f_{n}\right)^{1 / 2}-E_{n}\left(f_{n}^{\prime}\right)^{1 / 2}\right|\left(E_{n}\left(f_{n}\right)^{1 / 2}+E_{n}\left(f_{n}^{\prime}\right)^{1 / 2}\right) \\
& \leq \sqrt{2} d_{\mathcal{M}_{n}}\left(f_{n}, f_{n}^{\prime}\right)\left(2 E_{n}\left(f_{n}\right)^{1 / 2}+\sqrt{2} d_{\mathcal{M}_{n}}\left(f_{n}, f_{n}^{\prime}\right)\right) \\
& <2 \epsilon\left(\sqrt{2} C^{1 / 2}+\epsilon\right)
\end{aligned}
$$

for every $n \in K_{\epsilon}$. Since $K_{\epsilon} \in \omega$, we have

$$
\left\{n \in \mathbb{N}:\left|E_{n}\left(f_{n}\right)-E_{n}\left(f_{n}^{\prime}\right)\right|<2 \epsilon\left(\sqrt{2} C^{1 / 2}+\epsilon\right)\right\} \in \omega
$$

for any $\epsilon>0$. This implies that $\omega-\lim _{n} E_{n}\left(f_{n}\right)=\omega-\lim _{n} E_{n}\left(f_{n}^{\prime}\right)$.

\section{Global Busemann nonpositive Curvature spaces}

In this section, we recall the definition and some properties of global Busemann nonpositive curvature spaces. A global Busemann nonpositive curvature space was introduced by Busemann in [4]. Most of what appears in this section is included in [8] and [13].

Let $(M, d)$ be a metric space. For a pair of points $p, q \in M$, a shortest geodesic joining $p$ to $q$ is an isometric embedding $c$ of a closed interval $[0, l]$ into $M$ such that $c(0)=p$ and $c(l)=q$. Note that $l=d(p, q)$. A metric space is called a geodesic space if any pair of points has a shortest geodesic joining them. 
Definition 4.1. A complete geodesic space $(N, d)$ is called a global Busemann nonpositive curvature space (global Busemann NPC space) if

$$
d\left(c_{1}\left(\frac{l_{1}}{2}\right), c_{2}\left(\frac{l_{2}}{2}\right)\right) \leq \frac{1}{2} d\left(c_{1}\left(l_{1}\right), c_{2}\left(l_{2}\right)\right)
$$

holds for all pairs of shortest geodesics $c_{i}:\left[0, l_{i}\right] \rightarrow N(i=1,2)$ satisfying $c_{1}(0)=$ $c_{2}(0)$. This inequality is called the Busemann NPC inequality.

Example 4.2. Hadamard spaces are global Busemann NPC spaces. Complete simply connected Riemannian manifolds with nonpositive sectional curvature, trees, Euclidean Bruhat-Tits buildings, and Hilbert spaces are Hadamard spaces. A strictly convex Banach space (i.e., a Banach space whose unit sphere does not have a nontrivial line segment) is also a global Busemann NPC space. In particular, the Lebesgue space $L_{p}([0,1])$ such that $p \neq 2$ is not Hadamard space, but, for $1<p<\infty$, it is a global Busemann NPC space. The difference between Hadamard spaces and global Busemann NPC spaces may be typically seen in that between Hilbert spaces and strictly convex Banach spaces.

The convexity of the distance function in the following theorem is the most important feature of global Busemann NPC spaces.

Theorem 4.3 (see [8]). Let $c_{0}:\left[0, l_{0}\right] \rightarrow N, c_{1}:\left[0, l_{1}\right] \rightarrow N$ be shortest geodesics in a global Busemann NPC space $(N, d)$. Then $d\left(c_{0}\left(l_{0} t\right), c_{1}\left(l_{1} t\right)\right)$ is a convex function on $[0,1]$.

From this theorem, any pair of points in a global Busemann NPC space can be joined by precisely one shortest geodesic. Hence global Busemann NPC spaces are simply connected. Furthermore, this theorem implies that the energy functional on a global Busemann NPC space is convex along a shortest geodesic.

The ultralimit of a sequence of geodesic spaces is a geodesic space. But the ultralimit of a sequence of global Busemann NPC spaces may not be a global Busemann NPC space. For example, Banach spaces $\left(\mathbb{R}^{2},\|\|_{p}\right)$ with $1<p<\infty$ are global Busemann NPC spaces where $\|u\|_{p}:=\left(\left|u^{1}\right|^{p}+\left|u^{2}\right|^{p}\right)^{1 / p}$ for $u=\left(u^{1}, u^{2}\right) \in \mathbb{R}^{2}$. But the $\omega-\lim _{n}\left(\mathbb{R}^{2},\|\|_{n}, 0\right)$ is not a global Busemann NPC space. Indeed, for $u=(1,0), v=(2,0), w=(0,1) \in \mathbb{R}^{2}$, we have

$$
\begin{aligned}
\|u-v\|_{n} & =\left(\left|u^{1}-v^{1}\right|^{n}+\left|u^{2}-v^{2}\right|^{n}\right)^{1 / n}=1, \\
\|u-w\|_{n} & =\left(\left|u^{1}-w^{1}\right|^{n}+\left|u^{2}-w^{2}\right|^{n}\right)^{1 / n}=2^{1 / n}, \\
\|v-w\|_{n} & =\left(\left|v^{1}-w^{1}\right|^{n}+\left|v^{2}-w^{2}\right|^{n}\right)^{1 / n}=\left(2^{n}+1\right)^{1 / n}, \\
\left\|u-\frac{v+w}{2}\right\|_{n} & =\left(\left|u^{1}-\frac{v^{1}+w^{1}}{2}\right|^{n}+\left|u^{2}-\frac{v^{2}+w^{2}}{2}\right|^{n}\right)^{1 / n}=\frac{1}{2} .
\end{aligned}
$$

Thus, for $\left(u_{n}\right),\left(v_{n}\right)$ and $\left(w_{n}\right) \in \prod_{n \in \mathbb{N}} \mathbb{R}^{2}$ with $u_{n} \equiv u, v_{n} \equiv v$, and $w_{n} \equiv w$, we have $\left\|u_{\omega}-v_{\omega}\right\|_{\omega}=1,\left\|u_{\omega}-w_{\omega}\right\|_{\omega}=1$, and $\left\|v_{\omega}-w_{\omega}\right\|_{\omega}=2$. On the other hand, $u_{\omega} \neq\left(v_{\omega}+w_{\omega}\right) / 2$ holds. Since the line segment from $u$ to $w$ is a shortest geodesic, the line segment from $v_{\omega}$ to $w_{\omega}$ is also a shortest geodesic. Since $\left\|u_{\omega}-v_{\omega}\right\|_{\omega}+\| u_{\omega}-$ $w_{\omega}\left\|_{\omega}=\right\| v_{\omega}-w_{\omega} \|_{\omega}$, the path from $v_{\omega}$ through $u_{\omega}$ to $w_{\omega}$ whose part between $v_{\omega}$ and $u_{\omega}$ is a line segment and the part between $u_{\omega}$ and $w_{\omega}$ is also a line segment is another shortest geodesic. Hence in the ultralimit $\omega-\lim _{n}\left(\mathbb{R}^{2},\|\|_{n}, 0\right)$ the shortest 
geodesic between two points is not unique. Therefore the ultralimit is not a global Busemann NPC space.

\section{Absolute gradient}

In this section, we define the absolute gradient and investigate its properties. Let $\left(\mathcal{M}, d_{\mathcal{M}}\right)$ be a complete geodesic space, and $F: \mathcal{M} \rightarrow[0, \infty)$ a lower semicontinuous convex function. Here 'convex' means convex along all shortest geodesics.

Definition 5.1. We define the absolute gradient $\left|\nabla_{-} F\right|$ of $F$ at $f \in \mathcal{M}$ by

$$
\left|\nabla_{-} F\right|(f):=\max \left\{\limsup _{g \rightarrow f, g \in \mathcal{M}} \frac{F(f)-F(g)}{d_{\mathcal{M}}(f, g)}, 0\right\} .
$$

For Hadamard spaces, the following Proposition 5.2 and Proposition 5.3 are proved by Mayer in [11]. The proofs are valid for complete geodesic spaces.

Proposition 5.2. For $f \in \mathcal{M}$, we have

$$
\left|\nabla_{-} F\right|(f)=\max \left\{\sup _{g \neq f, g \in \mathcal{M}} \frac{F(f)-F(g)}{d_{\mathcal{M}}(f, g)}, 0\right\} .
$$

Proposition 5.3. Suppose $\left|\nabla_{-} F\right|$ is bounded. Then we have the following:

(i) $\left|\nabla_{-} F\right|$ is lower semicontinuous.

(ii) A point $f$ minimizes $F$ if and only if $\left|\nabla_{-} F\right|(f)=0$ holds.

Note that, as we saw in the proof of Lemma 3.4 the energy functional $E$ satisfies

$$
|E(f)-E(g)| \leq 2 d_{\mathcal{M}}(f, g)\left(\sqrt{2} E(f)^{1 / 2}+d_{\mathcal{M}}(f, g)\right) .
$$

This implies

$$
\left|\nabla_{-} E\right|(f) \leq \limsup _{g \rightarrow f, g \in \mathcal{M}} \frac{|E(f)-E(g)|}{d_{\mathcal{M}}(f, g)} \leq 2 \sqrt{2} E(f)^{1 / 2}
$$

for all $f \in \mathcal{M}$. In particular, the absolute gradient $\left|\nabla_{-} E\right|$ is bounded.

In 11, Mayer constructed a gradient flow of a lower semicontinuous convex function $F$ on a Hadamard space. Although, on global Busemann NPC spaces, it seems that it is difficult to construct such a gradient flow by Mayer's method, we can prove the following:

\section{Lemma 5.4.}

$$
\inf _{f \in \mathcal{M}}\left|\nabla_{-} F\right|(f)=0 .
$$

Proof. Suppose that $C:=\inf _{f \in \mathcal{M}}\left|\nabla_{-} F\right|(f)$ is positive. Then

$$
C \leq \sup _{g \neq f, g \in \mathcal{M}} \frac{F(f)-F(g)}{d_{\mathcal{M}}(f, g)}
$$

holds for all $f \in \mathcal{M}$ by Proposition [5.2. In particular, $F(f)>0$ for all $f \in \mathcal{M}$. Take $f_{0} \in \mathcal{M}$ and $0<\epsilon<1$ arbitrarily and set

$$
A_{0}:=\left\{g \in \mathcal{M} \backslash\left\{f_{0}\right\}:(1-\epsilon) C \leq \frac{F\left(f_{0}\right)-F(g)}{d_{\mathcal{M}}\left(f_{0}, g\right)}\right\} .
$$

By the definition of $C, A_{0}$ is not empty. 
If $\inf _{g \in A_{0}} F(g) \neq 0$, take $f_{1} \in A_{0}$ such that $F\left(f_{1}\right) \leq(1+\epsilon) \inf _{g \in A_{0}} F(g)$ and set

$$
A_{1}:=\left\{g \in \mathcal{M} \backslash\left\{f_{1}\right\}:(1-\epsilon) C \leq \frac{F\left(f_{1}\right)-F(g)}{d_{\mathcal{M}}\left(f_{1}, g\right)}\right\} .
$$

By the definition of $C, A_{1}$ is not empty. Since $f_{1} \in A_{0}$, for any $g \in A_{1}$, we have

$$
\begin{aligned}
\frac{F\left(f_{0}\right)-F(g)}{d_{\mathcal{M}}\left(f_{0}, g\right)} & \geq \frac{\left(F\left(f_{0}\right)-F\left(f_{1}\right)\right)+\left(F\left(f_{1}\right)-F(g)\right)}{d_{\mathcal{M}}\left(f_{0}, f_{1}\right)+d_{\mathcal{M}}\left(f_{1}, g\right)} \\
& \geq \frac{(1-\epsilon) C d_{\mathcal{M}}\left(f_{0}, f_{1}\right)+(1-\epsilon) C d_{\mathcal{M}}\left(f_{1}, g\right)}{d_{\mathcal{M}}\left(f_{0}, f_{1}\right)+d_{\mathcal{M}}\left(f_{1}, g\right)} \\
& =(1-\epsilon) C .
\end{aligned}
$$

Hence $g \in A_{0}$ holds, that is, $A_{1} \subset A_{0}$. Thus $\inf _{g \in A_{1}} F(g) \neq 0$ holds. Inductively, for each $i \in \mathbb{N}$, we can take $f_{i} \in A_{i-1}$ such that $F\left(f_{i}\right) \leq\left(1+\epsilon^{i}\right) \inf _{g \in A_{i-1}} F(g)$, and set

$$
A_{i}:=\left\{g \in \mathcal{M} \backslash\left\{f_{i}\right\}:(1-\epsilon) C \leq \frac{F\left(f_{i}\right)-F(g)}{d_{\mathcal{M}}\left(f_{i}, g\right)}\right\} \neq \emptyset .
$$

Then we have $A_{i} \subset A_{i-1}$ for each $i \in \mathbb{N}$ and $\inf _{g \in A_{i}} F(g) \neq 0$. Thus for $g \in A_{i}$ we have

$$
\begin{aligned}
d_{\mathcal{M}}\left(f_{i}, g\right) & \leq \frac{F\left(f_{i}\right)-F(g)}{(1-\epsilon) C} \\
& \leq \frac{\left(1+\epsilon^{i}\right) \inf _{g \in A_{i-1}} F(g)-\inf _{g \in A_{i}} F(g)}{(1-\epsilon) C} \\
& \leq \frac{\left(1+\epsilon^{i}\right) \inf _{g \in A_{i-1}} F(g)-\inf _{g \in A_{i-1}} F(g)}{(1-\epsilon) C} \\
& =\frac{\epsilon^{i} \inf _{g \in A_{i-1}} F(g)}{(1-\epsilon) C} .
\end{aligned}
$$

Since $f_{i} \in A_{i-1}$ and $F\left(f_{i}\right) \leq F\left(f_{i-1}\right)$ hold for each $i \in \mathbb{N}$, we have

$$
d_{\mathcal{M}}\left(f_{i}, g\right) \leq \frac{\epsilon^{i} F\left(f_{i}\right)}{(1-\epsilon) C} \leq \frac{\epsilon^{i} F\left(f_{0}\right)}{(1-\epsilon) C}
$$

for all $g \in A_{i}$. Therefore, for any $\epsilon^{\prime}>0$, there exists $i \in \mathbb{N}$ such that

$$
d_{\mathcal{M}}\left(f_{j}, f_{k}\right) \leq \operatorname{diam} A_{i} \leq 2 \frac{\epsilon^{i} F\left(f_{0}\right)}{(1-\epsilon) C}<\epsilon^{\prime}
$$

for every $j, k \geq i$. Since $\mathcal{M}$ is complete, $f_{i}$ converges to some $f_{\infty} \in \mathcal{M}$. Thus $\bigcap_{i=0}^{\infty}\left(A_{i} \cup\left\{f_{i}\right\}\right)$ is either an empty set or a one-point set $\left\{f_{\infty}\right\}$.

On the other hand, the function $1 / d_{\mathcal{M}}\left(f_{i}, g\right)$ is continuous on the open set $\mathcal{M} \backslash\left\{f_{i}\right\}$, and $-F(g)$ is upper semicontinuous on $\mathcal{M}$. Thus

$$
F_{i}^{\prime}(g):=\frac{F\left(f_{i}\right)-F(g)}{d_{\mathcal{M}}\left(f_{i}, g\right)}
$$

is upper semicontinuous on $\mathcal{M} \backslash\left\{f_{i}\right\}$. Hence $\left\{g \in \mathcal{M} \backslash\left\{f_{i}\right\}: F_{i}^{\prime}(g)<r\right\}$ is open for any $r>0$. This implies that

$$
\begin{aligned}
\left\{g \in \mathcal{M} \backslash\left\{f_{i}\right\}: F_{i}^{\prime}(g)<(1-\epsilon) C\right\} & =\mathcal{M} \backslash\left(\left\{g \in \mathcal{M}:(1-\epsilon) C \leq F_{i}^{\prime}(g)\right\} \cup\left\{f_{i}\right\}\right) \\
& =\mathcal{M} \backslash\left(A_{i} \cup\left\{f_{i}\right\}\right)
\end{aligned}
$$


is open, that is, $A_{i} \cup\left\{f_{i}\right\}$ is closed. Hence $f_{\infty} \in A_{i} \cup\left\{f_{i}\right\}$ for every $i$. This implies that $\bigcap_{i=0}^{\infty}\left(A_{i} \cup\left\{f_{i}\right\}\right)=\left\{f_{\infty}\right\}$. However, by assumption, there exists $g_{0} \in \mathcal{M} \backslash\left\{f_{\infty}\right\}$ such that

$$
(1-\epsilon) C \leq \frac{F\left(f_{\infty}\right)-F\left(g_{0}\right)}{d_{\mathcal{M}}\left(f_{\infty}, g_{0}\right)} .
$$

Since $f_{\infty} \in A_{i} \cup\left\{f_{i}\right\}$ and $A_{i+1} \cup\left\{f_{i+1}\right\} \subset A_{i}$, we have $f_{\infty} \in A_{i}$ for each $i$. Hence $F\left(g_{0}\right)<F\left(f_{\infty}\right)<F\left(f_{i}\right)$ for each $i$. Thus we have $f_{i} \neq g_{0}$ and

$$
\begin{aligned}
\frac{F\left(f_{i}\right)-F\left(g_{0}\right)}{d_{\mathcal{M}}\left(f_{i}, g_{0}\right)} & \geq \frac{\left(F\left(f_{i}\right)-F\left(f_{\infty}\right)\right)+\left(F\left(f_{\infty}\right)-F\left(g_{0}\right)\right)}{d_{\mathcal{M}}\left(f_{i}, f_{\infty}\right)+d_{\mathcal{M}}\left(f_{\infty}, g_{0}\right)} \\
& \geq \frac{(1-\epsilon) C d_{\mathcal{M}}\left(f_{i}, f_{\infty}\right)+(1-\epsilon) C d_{\mathcal{M}}\left(f_{\infty}, g_{0}\right)}{d_{\mathcal{M}}\left(f_{i}, f_{\infty}\right)+d_{\mathcal{M}}\left(f_{\infty}, g_{0}\right)} \\
& =(1-\epsilon) C
\end{aligned}
$$

for every $i$. This implies that $g_{0} \in \bigcap_{i=1}^{\infty}\left(A_{i} \cup\left\{f_{i}\right\}\right)=\left\{f_{\infty}\right\}$, that is, $f_{\infty}=g_{0}$. This contradicts $g_{0} \in \mathcal{M} \backslash\left\{f_{\infty}\right\}$.

If $\inf _{g \in A_{0}} F(g)=0$, take $f_{1}^{\prime} \in A_{0}$ such that $F\left(f_{1}^{\prime}\right) \leq \epsilon F\left(f_{0}\right)$, and set

$$
A_{1}^{\prime}:=\left\{g \in \mathcal{M} \backslash\left\{f_{1}^{\prime}\right\}:(1-\epsilon) C \leq \frac{F\left(f_{1}^{\prime}\right)-F(g)}{d_{\mathcal{M}}\left(f_{1}^{\prime}, g\right)}\right\} .
$$

By the definition of $C, A_{1}^{\prime}$ is not empty and is a subset of $A_{0}$. If $\inf _{g \in A_{1}^{\prime}} F(g) \neq 0$, then we can deduce a contradiction as in the case of $\inf _{g \in A_{0}} F(g) \neq 0$. Otherwise we take $f_{2}^{\prime} \in A_{1}^{\prime}$ such that $F\left(f_{2}^{\prime}\right) \leq \epsilon F\left(f_{1}^{\prime}\right)$, and set

$$
A_{2}^{\prime}:=\left\{g \in \mathcal{M} \backslash\left\{f_{2}^{\prime}\right\}:(1-\epsilon) C \leq \frac{F\left(f_{2}^{\prime}\right)-F(g)}{d_{\mathcal{M}}\left(f_{2}^{\prime}, g\right)}\right\} .
$$

Inductively, for each $i \in \mathbb{N}$, if $\inf _{g \in A_{i-1}^{\prime}} F(g) \neq 0$, then we can deduce a contradiction as in the case of $\inf _{g \in A_{0}} F(g) \neq 0$. Otherwise we take $f_{i}^{\prime} \in A_{i-1}$ such that $F\left(f_{i}^{\prime}\right) \leq \epsilon F\left(f_{i-1}^{\prime}\right)$, and set

$$
A_{i}^{\prime}:=\left\{g \in \mathcal{M} \backslash\left\{f_{i}^{\prime}\right\}:(1-\epsilon) C \leq \frac{F\left(f_{i}^{\prime}\right)-F(g)}{d_{\mathcal{M}}\left(f_{i}^{\prime}, g\right)}\right\} \neq \emptyset .
$$

Then we have $A_{i}^{\prime} \subset A_{i-1}^{\prime}$ for each $i \in \mathbb{N}$. Thus for $g \in A_{i}^{\prime}$ we have

$$
d_{\mathcal{M}}\left(f_{i}^{\prime}, g\right) \leq \frac{F\left(f_{i}^{\prime}\right)-F(g)}{(1-\epsilon) C} \leq \frac{\epsilon F\left(f_{i-1}^{\prime}\right)}{(1-\epsilon) C} \leq \frac{\epsilon^{i} F\left(f_{0}^{\prime}\right)}{(1-\epsilon) C} .
$$

Hence $f_{i}^{\prime}$ converges to some $f_{\infty}^{\prime} \in \mathcal{M}$ and $\bigcap_{i=0}^{\infty}\left(A_{i}^{\prime} \cup\left\{f_{i}^{\prime}\right\}\right)=\left\{f_{\infty}^{\prime}\right\}$. Therefore we can deduce a contradiction as in the case of $f_{\infty}$.

Corollary 5.5. There exists a sequence in $\mathcal{M}$ such that the absolute gradient approaches 0 . If the sequence converges to a point, then the point minimizes $F$.

Proof. By Lemma 5.4, the existence of such a sequence $f_{n} \in \mathcal{M}$ is obvious. By Proposition [5.3, $\left|\nabla_{-} F\right|$ is lower semicontinuous. Hence we have

$$
\left|\nabla_{-} F\right|\left(f_{\infty}\right) \leq \lim _{n \rightarrow \infty}\left|\nabla_{-} F\right|\left(f_{n}\right)=0 .
$$




\section{The fixed-Point Property for a GLOBAl Busemann NPC SPACE}

In this section, we prove Theorem 1 and Theorem 2 . Let $\Gamma$ be a finitely generated group, $S$ a finite generating subset, and $m$ a weight as in Section 3 .

Proposition 6.1. Let $\omega$ be a nonprincipal ultrafilter, $\left(N_{n}, d_{n}\right)$ a sequence of global Busemann NPC spaces, and $\rho_{n}: \Gamma \rightarrow \operatorname{Isom}\left(N_{n}, d_{n}\right)$ a sequence of homomorphisms. Denote by $E_{n}$ the energy functional on the set of all $\rho_{n}$-equivariant maps from $\Gamma$ into $N_{n}$. Take a $\rho_{n}$-equivariant map $f_{n}$ arbitrarily for each $n$, and set $f_{n}(e)$ as a base point of $\left(N_{n}, d_{n}\right)$. Suppose that $E_{n}\left(f_{n}\right)$ is bounded independently of $n$ and that $\left|\nabla_{-} E_{n}\right|\left(f_{n}\right) \rightarrow 0$ as $n \rightarrow \infty$. Then there exists a limit map $\omega-\lim _{n} f_{n}$, which is a harmonic $\rho_{\omega}$-equivariant map.

Proof. Since $E_{n}\left(f_{n}\right)$ is uniformly bounded independently of $n$, there exists $C>0$ such that $d_{n}\left(f_{n}(e), f_{n}(\gamma)\right)<C$ independently of $n$ and $\gamma \in S$. Hence we can define the ultralimit $\rho_{\omega}$ of $\rho_{n}$ and $\rho_{\omega}$-equivariant map $f_{\omega}(\gamma)=\omega-\lim _{n} f_{n}(\gamma)$ by Lemma 3.2 and Lemma 3.3. We show that this limit map $f_{\omega}$ is harmonic. Take $g \in \mathcal{M}_{\omega}$ arbitrarily, and let $\left(g_{n}\right) \in \mathcal{M}_{\infty}$ be a representative of $g$. Then $d_{\mathcal{M}_{n}}\left(f_{n}, g_{n}\right)=$ $d\left(f_{n}(e), g_{n}(e)\right)$ is uniformly bounded by some constant $\hat{C}$ independent of $n$. By Proposition 5.2, we have

$$
\left|\nabla_{-} E_{n}\right|\left(f_{n}\right) \geq \sup _{g \neq f_{n}, g \in \mathcal{M}_{n}} \frac{E_{n}\left(f_{n}\right)-E_{n}(g)}{d_{\mathcal{M}_{n}}\left(f_{n}, g\right)} \geq \frac{E_{n}\left(f_{n}\right)-E_{n}\left(g_{n}\right)}{d_{\mathcal{M}_{n}}\left(f_{n}, g_{n}\right)}
$$

By the assumption that $\left|\nabla_{-} E_{n}\right|\left(f_{n}\right) \rightarrow 0$ as $n \rightarrow \infty$, we get

$$
E_{n}\left(f_{n}\right)-E_{n}\left(g_{n}\right) \leq d_{\mathcal{M}_{n}}\left(f_{n}, g_{n}\right)\left|\nabla_{-} E_{n}\right|\left(f_{n}\right) \leq \hat{C}\left|\nabla_{-} E_{n}\right|\left(f_{n}\right) \rightarrow 0
$$

as $n \rightarrow \infty$. By Lemma 3.4 we have

$$
E_{\omega}\left(f_{\omega}\right)-E_{\omega}(g)=\omega-\lim _{n}\left(E_{n}\left(f_{n}\right)-E_{n}\left(g_{n}\right)\right) \leq \hat{C} \omega-\lim _{n}\left|\nabla_{-} E_{n}\right|\left(f_{n}\right)=0 .
$$

Therefore, we obtain $E_{\omega}\left(f_{\omega}\right) \leq E_{\omega}(g)$ for all $g \in \mathcal{M}_{\omega}$, that is, $f_{\omega}$ is harmonic.

Using this proposition, we deduce the following two main theorems.

Theorem 1. Let $(N, d)$ be a global Busemann $N P C$ space, $\rho: \Gamma \rightarrow \operatorname{Isom}(N, d)$ a homomorphism. Denote by $E$ the energy functional on the space of $\rho$-equivariant maps. Take a sequence of $\rho$-equivariant maps $f_{n}$ satisfying $\left|\nabla_{-} E\right|\left(f_{n}\right) \rightarrow 0$ as $n \rightarrow \infty$. If there exists a positive constant $C$ such that

$$
\left|\nabla_{-} E\right|\left(f_{n}\right)^{2} \geq C E\left(f_{n}\right)
$$

for every $n$, then, for any nonprincipal ultrafilter $\omega$ on $\mathbb{N}$, there exists a fixed point of $\rho_{\omega}$ in $\left(N_{\omega}, d_{\omega}\right):=\omega-\lim _{n}\left(N, d, f_{n}(e)\right)$.

Proof. Set $\left(N_{n}, d_{n}\right):=(N, d)$, and denote by $E_{n}(=E)$ the energy functional for $\rho$-equivariant maps from $\Gamma$ into $N_{n}$. By assumption, we have

$$
C E_{n}\left(f_{n}\right) \leq\left|\nabla_{-} E_{n}\right|\left(f_{n}\right)^{2} \rightarrow 0
$$

as $n \rightarrow \infty$. Hence $E_{n}\left(f_{n}\right) \rightarrow 0$ as $n \rightarrow \infty$. This implies that $E_{n}\left(f_{n}\right)$ is uniformly bounded independent of $n$. By Proposition 6.1 for any nonprincipal ultrafilter $\omega$, there exists a $\rho_{\omega}$-equivariant harmonic map $f_{\omega}:=\omega-\lim _{n} f_{n}$ such that $E_{\omega}\left(f_{\omega}\right)=0$. Therefore $f_{\omega}(e)$ is a fixed point of $\rho_{\omega}(\Gamma)$. 
Theorem 2. Let $\mathcal{L}$ be a family of global Busemann NPC spaces which is stable under ultralimit. Suppose that, for any $(N, d) \in \mathcal{L}$ and $\rho: \Gamma \rightarrow \operatorname{Isom}(N, d)$, there exists a fixed point of $\rho(\Gamma)$. Then there exists a positive constant $C$ such that

$$
\left|\nabla_{-} E_{N, \rho}\right|(f)^{2} \geq C E_{N, \rho}(f)
$$

holds for all $(N, d) \in \mathcal{L}$, homomorphisms $\rho: \Gamma \rightarrow \operatorname{Isom}(N, d)$ and $\rho$-equivariant maps $f$, where $E_{N, \rho}$ denotes the energy functional for $\rho$-equivariant maps. The constant $C=C(\Gamma, \mathcal{L})$ should be independent of $(N, d), \rho$, and $f$.

Proof. The proof is by contradiction. Assume that the assertion is false. Then, for each $n$, there exist $\left(N_{n}, d_{n}\right) \in \mathcal{L}$, a homomorphism $\rho_{n}: \Gamma \rightarrow \operatorname{Isom}\left(N_{n}, d_{n}\right)$ and a $\rho_{n}$-equivariant map $f_{n}: \Gamma \rightarrow N_{n}$ such that

$$
\left|\nabla_{-} E_{N_{n}, \rho_{n}}\right|\left(f_{n}\right)^{2}<\frac{1}{n} E_{N_{n}, \rho_{n}}\left(f_{n}\right) .
$$

In particular, $E_{N_{n}, \rho_{n}}\left(f_{n}\right)>0$ holds for every $n$. Because of the assumption, there exists a $\rho_{n}$-equivariant map $g_{n}: \Gamma \rightarrow N_{n}$ for each $n$ which satisfies $E_{N_{n}, \rho_{n}}\left(g_{n}\right)=0$. Thus $f_{n}$ does not minimize $E_{N_{n}, \rho_{n}}$. By Proposition 5.3, we have $\left|\nabla_{-} E_{N_{n}, \rho_{n}}\right|\left(f_{n}\right)>$ 0 for every $n$. Set $\left(N_{n}^{\prime}, d_{n}^{\prime}, f_{n}(e)\right):=\left(N_{n}, E_{N_{n}, \rho_{n}}\left(f_{n}\right)^{-1 / 2} d_{n}, f_{n}(e)\right)$. We can regard $\rho_{n}: \Gamma \rightarrow \operatorname{Isom}\left(N_{n}, d_{n}\right)$ as a homomorphism $\rho_{n}: \Gamma \rightarrow \operatorname{Isom}\left(N_{n}^{\prime}, d_{n}^{\prime}\right)$. Moreover, we have $E_{N_{n}^{\prime}, \rho_{n}}\left(f_{n}\right)=1$ for every $n$; hence $E_{N_{n}^{\prime}, \rho_{n}}\left(f_{n}\right)$ is bounded independent of $n$. We have $\left|\nabla_{-} E_{N_{n}^{\prime}, \rho_{n}}\right|\left(f_{n}\right)^{2}<1 / n$ for every $n$. Take a nonprincipal ultrafilter $\omega$ so that $\left(N_{\omega}^{\prime}, d_{\omega}^{\prime}\right) \in \mathcal{L}$. By Proposition 6.1, the $\rho_{\omega}$-equivariant map $f_{\omega}:=\omega-\lim _{n} f_{n}$ minimizes $E_{N_{\omega}^{\prime}, \rho_{\omega}}$. On the other hand, we have $E_{N_{\omega}^{\prime}, \rho_{\omega}}\left(f_{\omega}\right)=1$. This contradicts our assumption.

We give some examples of a family of global Busemann NPC spaces which is stable under ultralimit.

Example 6.2. For a Banach space $(B,\|\|)$, the function

$$
\delta_{B}(\epsilon):=\inf \{1-\|u+v\| / 2:\|u\|,\|v\| \leq 1,\|u-v\| \geq \epsilon\}
$$

is called the modulus of convexity of $B$. A Banach space $B$ is said to be uniformly convex if $\delta_{B}(\epsilon)>0$ for any $\epsilon>0$. Uniformly convex Banach spaces are strictly convex Banach spaces. For example, the Lebesgue space $L_{p}([0,1])$ satisfies

$$
\delta_{L_{p}([0,1])}(\epsilon)= \begin{cases}(p-1) \epsilon^{2} / 8+o\left(\epsilon^{2}\right) & \text { if } 1<p \leq 2 \\ \epsilon^{p} /\left(p 2^{p}\right)+o\left(\epsilon^{p}\right) & \text { if } 2 \leq p<\infty\end{cases}
$$

see [3. Let $\delta:[0,2] \rightarrow[0,1]$ be a left continuous, monotone increasing function which satisfies $\delta(0)=0$ and $\delta(\epsilon)>0$ for any $\epsilon>0$. Then the family $\mathcal{L}_{\delta}$ that consists of all Banach spaces $B$ with $\delta_{B}(\epsilon) \geq \delta(\epsilon)$ for any $\epsilon>0$ is an example of a family of global Busemann NPC spaces which is stable under ultralimit, because we have the following: For a nonprincipal ultrafilter $\omega$, a sequence $\left(B_{n},\|\|_{n}\right) \in \mathcal{L}_{\delta}$, $r_{n}>0$ and $o_{n} \in B_{n}$, set $\left(B_{\omega},\|\|_{\omega}\right)=\omega-\lim _{n}\left(B_{n}, r_{n}\|\|_{n}, o_{n}\right)$. Take $0<\epsilon \leq 2$ and $u, v \in B_{\omega}$ such that $\|u\|_{\omega}=\|v\|_{\omega}=1$ and $\|u-v\|_{\omega} \geq \epsilon$. For representatives $\left(u_{n}\right)$, $\left(v_{n}\right) \in B_{\infty}$ and for any $0<\eta<\epsilon$, we have

$$
\left\{n \in \mathbb{N}:\left|r_{n}\left\|u_{n}\right\|_{n}-1\right|<\eta,\left|r_{n}\left\|v_{n}\right\|_{n}-1\right|<\eta, r_{n}\left\|u_{n}-v_{n}\right\|_{n}>\epsilon-\eta\right\} \in \omega .
$$

Hence we obtain

$$
\left\{n \in \mathbb{N}: \frac{r_{n}\left\|u_{n}\right\|_{n}}{1+\eta}<1, \frac{r_{n}\left\|v_{n}\right\|_{n}}{1+\eta}<1, \frac{r_{n}\left\|u_{n}-v_{n}\right\|_{n}}{1+\eta}>\frac{\epsilon-\eta}{1+\eta}\right\} \in \omega .
$$


This implies that

$$
\left\{n \in \mathbb{N}: \frac{r_{n}\left\|u_{n}+v_{n}\right\|_{n}}{2(1+\eta)} \leq 1-\delta_{B_{n}}\left(\frac{\epsilon-\eta}{1+\eta}\right)\right\} \in \omega
$$

for any $0<\eta<\epsilon$. Therefore we have

$$
\frac{\|u+v\|_{\omega}}{2} \leq \inf _{\eta>0}(1+\eta)\left(1-\delta\left(\frac{\epsilon-\eta}{1+\eta}\right)\right)=1-\delta(\epsilon),
$$

that is, $\delta_{B_{\omega}}(\epsilon) \geq \delta(\epsilon)$ for any $\epsilon>0$.

Example 6.3. Fix $k>0$ arbitrarily. Let $\mathcal{L}_{k}$ be the family of global Busemann NPC spaces $(N, r d)$, where $r>0$ and $(N, d)$ are global Busemann NPC spaces with the following condition: For any point $p \in N$ and geodesic $c:[0, l] \rightarrow N$,

$$
k d(p, c(t l))^{2} \leq(1-t) d(p, c(0))^{2}+t d(p, c(l))^{2}-(1-t) t d(c(0), c(l))^{2}
$$

holds for all $0 \leq t \leq 1$. Then the family $\mathcal{L}_{k}$ is stable under ultralimit. Note that the family $\mathcal{L}_{1}$ consists of all Hadamard spaces.

The reason why $\mathcal{L}_{k}$ is stable under ultralimit is the following: For a nonprincipal ultrafilter $\omega$ and sequences $\left(N_{n}, d_{n}\right) \in \mathcal{L}_{k}$ and $o_{n} \in N_{n}$, set $\left(N_{\omega}, d_{\omega}\right)=\omega$ $\lim _{n}\left(N_{n}, d_{n}, o_{n}\right)$. Take arbitrary $p, q \in N_{\omega}$, and let $\left(p_{n}\right),\left(q_{n}\right) \in N_{\infty}$ be their representatives respectively. For a sequence of shortest geodesics $c_{n}:\left[0, l_{n}\right] \rightarrow N_{n}$ from $p_{n}$ to $q_{n}$, the limit map $c_{\omega}:\left[0, l_{\omega}\right] \rightarrow N_{\omega}$ defined by $c_{\omega}(t):=\omega-\lim _{n} c_{n}\left(t l_{n} / l_{\omega}\right)$ is a shortest geodesic from $p$ to $q$. Take a shortest geodesic $\tilde{c}:[0, l] \rightarrow N_{\omega}$ from $p$ to $q$, and representations $\left(\tilde{c}_{n}(t)\right) \in N_{\infty}$ of $\tilde{c}(t)$ for each $t \in[0, l]$, where $l=l_{\omega}$. By the definition of $\mathcal{L}_{k}$, we have

$$
\begin{aligned}
& k d_{n}\left(\tilde{c}_{n}(t l), c_{n}\left(t l_{n}\right)\right)^{2} \\
\leq & (1-t) d_{n}\left(\tilde{c}_{n}(t l), c_{n}(0)\right)^{2}+t d_{n}\left(\tilde{c}_{n}(t l), c_{n}\left(l_{n}\right)\right)^{2}-(1-t) t d_{n}\left(c_{n}(0), c_{n}\left(l_{n}\right)\right)^{2} \\
= & (1-t)\left\{d_{n}\left(\tilde{c}_{n}(t l), c_{n}(0)\right)^{2}-\left(t d_{n}\left(c_{n}(0), c_{n}\left(l_{n}\right)\right)\right)^{2}\right\} \\
& \quad+t\left\{d_{n}\left(\tilde{c}_{n}(t l), c_{n}\left(l_{n}\right)\right)^{2}-\left((1-t) d_{n}\left(c_{n}(0), c_{n}\left(l_{n}\right)\right)\right)^{2}\right\} \\
= & (1-t)\left\{d_{n}\left(\tilde{c}_{n}(t l), p_{n}\right)^{2}-\left(t l_{n}\right)^{2}\right\}+t\left\{d_{n}\left(\tilde{c}_{n}(t l), q_{n}\right)^{2}-\left((1-t) l_{n}\right)^{2}\right\}
\end{aligned}
$$

for any $0 \leq t \leq 1$. Hence we obtain

$$
\begin{aligned}
k d_{\omega}\left(\tilde{c}(t l), c_{\omega}\left(t l_{\omega}\right)\right)^{2} & \leq(1-t)\left\{(t l)^{2}-\left(t l_{\omega}\right)^{2}\right\}+t\left\{((1-t) l)^{2}-\left((1-t) l_{\omega}\right)^{2}\right\} \\
& =0
\end{aligned}
$$

for all $0 \leq t \leq 1$. Therefore the shortest geodesic from $p$ to $q$ is unique, and it is the ultralimit of shortest geodesics. Hence the Busemann NPC inequalities for $\left(N_{n}, d_{n}\right)$ imply the Busemann NPC inequality for $\left(N_{\omega}, d_{\omega}\right)$. By a similar argument, for any point $p \in N_{\omega}$ and geodesic $c:[0, l] \rightarrow N_{\omega}$, we have

$$
k d_{\omega}(p, c(t))^{2} \leq(1-t) d_{\omega}(p, c(0))^{2}+t d_{\omega}(p, c(l))^{2}-(1-t) t d_{\omega}(c(0), c(l))^{2}
$$

for all $0 \leq t \leq l$.

Example 6.4. Fix $k>0$ arbitrarily. Let $\mathcal{L}^{\prime}{ }_{k}$ be a family of global Busemann NPC spaces $(N, r d)$ where $r>0$ and $(N, d)$ are global Busemann NPC spaces $(N, d)$ with the following condition: For any point $p \in N$ and geodesic $c:[0, l] \rightarrow N$,

$$
d(p, c(t))^{2} \leq(1-t) d(p, c(0))^{2}+t d(p, c(l))^{2}-k(1-t) t d(c(0), c(l))^{2}
$$


holds for all $0 \leq t \leq 1$. The metric space satisfying this inequality was introduced by Ohta in [12. This inequality induces the inequality in the definition of $\mathcal{L}_{k}$; see 12. The family $\mathcal{L}^{\prime}{ }_{k}$ is also stable under ultralimit. The proof is the same as that for $\mathcal{L}_{k}$.

\section{The FIXED-POINT PROPERTY FOR BANACH SPACES}

In this section, we review the definitions of the properties $\left(F_{B}\right),\left(T_{B}\right),\left(E_{B}\right)$ and $\left(E_{B}\right)^{\prime}$, and show Theorem 3 and Proposition 4.

Let $\Gamma$ be a finitely generated group, $S$ a finite generating subset, and $m$ a weight as in Section 3. Denote by $O(B)$ the full linear isometry group of a Banach space $(B,\|\|)$, and by $\operatorname{Isom}(B)$ the full affine isometry group of $(B,\|\|)$. A linear isometric $\Gamma$-representation $\pi$ on $B$ is a homomorphism $\pi: \Gamma \rightarrow O(B)$. Denote by $B^{\pi(\Gamma)}$ the closed subspace consisting of fixed vectors of $\pi$. We can define a linear isometric $\Gamma$-representation $\pi$ on $B / B^{\pi(\Gamma)}$ by $\pi(\gamma)[v]:=[\pi(\gamma) v]$ for each $[v] \in B / B^{\pi(\Gamma)}$ and $\gamma \in \Gamma$, where $[v] \in B / B^{\pi(\Gamma)}$ means the equivalence class of $v \in B$.

Similarly, an affine isometric $\Gamma$-representation $\rho$ on $B$ is a homomorphism $\rho$ : $\Gamma \rightarrow \operatorname{Isom}(B)$. An affine isometric $\Gamma$-representation $\rho$ on $B$ is written as $\rho(\gamma) v=$ $\rho_{0}(\gamma) v+o(\gamma)$ by a linear isometric $\Gamma$-representation $\rho_{0}$ on $B$ and a map $o: \Gamma \rightarrow$ $B$. We can define a linear isometric $\Gamma$-representation $\rho^{\prime}$ on $B^{\prime}:=B / B^{\rho_{0}(\Gamma)}$ by $\rho^{\prime}(\gamma)[v]:=[\rho(\gamma) v]=\rho_{0}^{\prime}(\gamma)[v]+[o(\gamma)]$ for each $[v] \in B^{\prime}$ and $\gamma \in \Gamma$.

Definition 7.1. (i) We say that $\Gamma$ has property $\left(F_{B}\right)$ if any affine isometric $\Gamma$ representation on $B$ has a fixed point.

(ii) We say that $\Gamma$ has property $\left(T_{B}\right)$ if, for any nontrivial linear isometric $\Gamma$ representation $\rho_{0}$, there exists a positive constant $C\left(\rho_{0}\right)$ such that

$$
\max _{\gamma \in S}\left\|[v]-\rho_{0}^{\prime}(\gamma)[v]\right\| \geq C\left(\rho_{0}\right)
$$

for all $[v] \in B / B^{\pi(\Gamma)}$ satisfying $\|[v]\|=1$.

(iii) We say that $\Gamma$ has property $\left(E_{B}\right)$ if there exists a positive constant $C(\rho)$ for any affine isometric $\Gamma$-representation $\rho$ such that

$$
\left|\nabla_{-} E_{\rho^{\prime}}\right|(f)^{2} \geq C(\rho) E_{\rho^{\prime}}(f)
$$

for all $\rho^{\prime}$-equivariant maps $f$.

Then we have the following theorem.

Theorem 3. If $\Gamma$ has property $\left(F_{B}\right)$, then it has property $\left(E_{B}\right)$.

Proof. Take an arbitrary affine isometric $\Gamma$-representation $\rho: \Gamma \rightarrow \operatorname{Isom}(B)$. Since $\Gamma$ has property $\left(F_{B}\right)$, there exists a fixed point $v_{0} \in B$ of $\rho(\Gamma)$. We can regard a fixed point $\left[v_{0}\right]$ of $\rho^{\prime}(\Gamma)$ as the origin of $B^{\prime}$. We recall that if $\Gamma$ has property $\left(F_{B}\right)$, then it has property $\left(T_{B}\right)$. Hence we have a positive constant $C\left(\rho^{\prime}\right)$ such that $\max _{\gamma \in S}\left\|[v]-\rho^{\prime}(\gamma)[v]\right\| \geq C\left(\rho^{\prime}\right)$ for all $[v] \in B^{\prime}$ satisfying $\|[v]\|=1$. If $\|f(e)\|=0$, then $E_{\rho^{\prime}}(f)=0 \leq\left|\nabla_{-} E_{\rho^{\prime}}\right|(f)$. Denote a linear action $I d-\rho^{\prime}(\gamma)$ by $T(\gamma)$ for each $\gamma \in S$, where $I d$ is the identity map on $B^{\prime}$. Then $E_{\rho^{\prime}}(f)=$ $\sum_{\gamma \in S} m(\gamma)\|T(\gamma) f(e)\|^{2} / 2$. Set $g_{\epsilon}(\gamma):=(1-\epsilon) f(\gamma)$ for $0<\epsilon<1$, which is a 
$\rho^{\prime}$-equivariant map. If $\|f(e)\| \neq 0$, then we have

$$
\begin{aligned}
& \left|\nabla_{-} E_{\rho^{\prime}}\right|(f) \\
\geq & \lim _{\epsilon \rightarrow 0} \frac{\sum_{\gamma \in S} m(\gamma)\|T(\gamma) f(e)\|^{2}-\sum_{\gamma \in S} m(\gamma)\left\|T(\gamma) g_{\epsilon}(e)\right\|^{2}}{2\left\|f(e)-g_{\epsilon}(e)\right\|} \\
= & \sum_{\gamma \in S} m(\gamma) \lim _{\epsilon \rightarrow 0}\left(\frac{\|T(\gamma) f(e)\|-\left\|T(\gamma) g_{\epsilon}(e)\right\|}{\left\|f(e)-g_{\epsilon}(e)\right\|} \frac{\|T(\gamma) f(e)\|+\left\|T(\gamma) g_{\epsilon}(e)\right\|}{2}\right) \\
= & \sum_{\gamma \in S} m(\gamma) \frac{\|T(\gamma) f(e)\|}{\|f(e)\|}\|T(\gamma) f(e)\| \\
\geq & \min _{\gamma \in S} m(\gamma) \max _{\gamma \in S} \frac{\|T(\gamma) f(e)\|}{\|f(e)\|} \max _{\gamma \in S}\|T(\gamma) f(e)\| \\
\geq & \min _{\gamma \in S} m(\gamma) C\left(\rho^{\prime}\right) \sum_{\gamma \in S} m(\gamma)\|T(\gamma) f(e)\| .
\end{aligned}
$$

Using the Hölder inequality, we have

$$
\begin{aligned}
E_{\rho^{\prime}}(f) & \leq \frac{1}{2}\left(\sum_{\gamma \in S} m(\gamma)^{2}\|T(\gamma) f(e)\|^{2}\right)^{1 / 2}\left(\sum_{\gamma \in S}\|T(\gamma) f(e)\|^{2}\right)^{1 / 2} \\
& \leq \frac{1}{2}\left(\sum_{\gamma \in S} m(\gamma)^{2}\|T(\gamma) f(e)\|^{2}\right)^{1 / 2}\left(\sum_{\gamma \in S} \frac{m(\gamma)^{2}}{\min _{\gamma \in S} m(\gamma)^{2}}\|T(\gamma) f(e)\|^{2}\right)^{1 / 2} \\
& \leq \frac{\sum_{\gamma \in S} m(\gamma)^{2}\|T(\gamma) f(e)\|^{2}}{2 \min _{\gamma \in S} m(\gamma)} \\
& \leq \frac{\left(\sum_{\gamma \in S} m(\gamma)\|T(\gamma) f(e)\|^{2}\right.}{2 \min _{\gamma \in S} m(\gamma)}
\end{aligned}
$$

Therefore we obtain $\left|\nabla_{-} E_{\rho^{\prime}}\right|(f)^{2} \geq 2 C\left(\rho^{\prime}\right)^{2} \min _{\gamma \in S} m(\gamma)^{3} E_{\rho^{\prime}}(f)$.

In order to state Proposition 4, we define a uniformly convex, uniformly smooth Banach space, which has good properties for investigating the absolute gradient.

Definition 7.2. (i) A Banach space $B$ is said to be uniformly convex if the modulus of convexity of $B$,

$$
\delta_{B}(\epsilon):=\inf \{1-\|u+v\| / 2:\|u\|,\|v\| \leq 1, \text { and }\|u-v\| \geq \epsilon\},
$$

is positive whenever $\epsilon>0$.

(ii) A Banach space $B$ is said to be uniformly smooth if the modulus of smoothness of $B$,

$$
\delta_{B}^{*}(\tau):=\sup \{\|u+v\| / 2+\|u-v\| / 2-1:\|u\| \leq 1 \text {, and }\|v\| \leq \tau\},
$$

satisfies $\delta_{B}^{*}(\tau) / \tau \rightarrow 0$ as $\tau \rightarrow 0$.

"Uniformly convex, uniformly smooth" is abbreviated to "ucus". Ucus Banach spaces are global Busemann NPC spaces. Examples of ucus Banach spaces are Hilbert spaces and the Lebesgue spaces $L_{p}([0,1])$ with $1<p<\infty$. Ucus Banach spaces $B$ are reflexive, and for any nonzero vector $v \in B$ there exists a unique functional $v^{*} \in B^{*}$ such that $\left\|v^{*}\right\|=1$ and $v^{*}(v)=\|v\|$. The duality map $v \in$ $B \backslash\{0\} \mapsto v^{*} \in B^{*}$ is uniformly continuous. For $0 \in B$, set $0^{*}=0 \in B^{*}$. See [3, App. A]. 
To prove Proposition 4, we prepare the following proposition.

Proposition 7.3. Let $B$ be a ucus Banach space and $\rho: \Gamma \rightarrow \operatorname{Isom}(B,\|\|)$ an affine isometric $\Gamma$-representation on $B$. Then we have

$$
\begin{aligned}
\left|\nabla_{-} E\right|(f) \geq & \sum_{\gamma \in S} m(\gamma)\|T(\gamma) f(e)\| \operatorname{Re}(T(\gamma) f(e))^{*} \frac{T_{0}(\gamma) v}{\|v\|} \\
& =2 \sum_{\gamma \in S} m(\gamma)\|T(\gamma) f(e)\| \operatorname{Re}(T(\gamma) f(e))^{*} \frac{v}{\|v\|}
\end{aligned}
$$

for all $\rho$-equivariant maps $f$ and nonzero vectors $v \in B$. Here $T(\gamma):=I d-\rho(\gamma)$, $T_{0}(\gamma):=I d-\rho_{0}(\gamma)$, and $\operatorname{Re} v^{*}(w)$ is the real part of $v^{*} w$ for $w \in B$. The function $\sum_{\gamma \in S} m(\gamma)\|T(\gamma) f(e)\| \operatorname{Re}(T(\gamma) f(e))^{*}$ is continuous on the space of $\rho$-equivariant maps.

Proof. Take an arbitrary $\rho$-equivariant map $f$ and a nonzero vector $v \in B$. Set $g_{\epsilon}(\gamma):=\rho(\gamma)(f(e)+\epsilon v)$ for $\epsilon>0$ and $\gamma \in \Gamma$. Computing as the proof of Theorem 3 , we have

$$
\left|\nabla_{-} E\right|(f) \geq \sum_{\gamma \in S} m(\gamma)\left(\frac{\|T(\gamma) f(e)\|+\left\|T(\gamma) g_{\epsilon}(e)\right\|}{2} \frac{\|T(\gamma) f(e)\|-\left\|T(\gamma) g_{\epsilon}(e)\right\|}{\epsilon\|v\|}\right)
$$

for any $\epsilon>0$. We get

$$
\begin{aligned}
\|T(\gamma) f(e)\|-\left\|T(\gamma) g_{\epsilon}(e)\right\| & \geq \operatorname{Re}\left(T(\gamma) g_{\epsilon}(e)\right)^{*}\left(T(\gamma) f(e)-T(\gamma) g_{\epsilon}(e)\right) \\
& =\epsilon \operatorname{Re}\left(T(\gamma) g_{\epsilon}(e)\right)^{*}\left(T_{0}(\gamma) v\right)
\end{aligned}
$$

for every $\gamma \in S$. On the other hand, since $T(\gamma)$ is continuous, $T(\gamma) g_{\epsilon}(e)$ converges to $T(\gamma) f(e)$ as $\epsilon \rightarrow 0$. Because the duality map is uniformly continuous, $\left(T(\gamma) g_{\epsilon}(e)\right)^{*}\left(T_{0}(\gamma) v\right)$ converges to $(T(\gamma) f(e))^{*}\left(T_{0}(\gamma) v\right)$ as $\epsilon \rightarrow 0$ for every $\gamma \in S$. Therefore we have

$$
\begin{aligned}
& \sum_{\gamma \in S} m(\gamma) \lim _{\epsilon \rightarrow 0}\left(\frac{\|T(\gamma) f(e)\|+\left\|T(\gamma) g_{\epsilon}(e)\right\|}{2} \frac{\|T(\gamma) f(e)\|-\left\|T(\gamma) g_{\epsilon}(e)\right\|}{\epsilon\|v\|}\right) \\
\geq & \sum_{\gamma \in S} m(\gamma)\|T(\gamma) f(e)\| \operatorname{Re}(T(\gamma) f(e))^{*} \frac{T_{0}(\gamma) v}{\|v\|} \\
= & \sum_{\gamma \in S} m(\gamma)\|T(\gamma) f(e)\|\left(\operatorname{Re}(T(\gamma) f(e))^{*} \frac{v}{\|v\|}-\operatorname{Re}(T(\gamma) f(e))^{*} \rho_{0}(\gamma) \frac{v}{\|v\|}\right) .
\end{aligned}
$$

Since $\rho_{0}(\gamma)$ is a linear isometry, we have $\left\|(T(\gamma) f(e))^{*} \rho_{0}(\gamma)\right\|=\left\|(T(\gamma) f(e))^{*}\right\|=1$ and

$$
\left((T(\gamma) f(e))^{*} \rho_{0}(\gamma)\right) \rho_{0}\left(\gamma^{-1}\right) T(\gamma) f(e)=\|T(\gamma) f(e)\|=\left\|\rho_{0}\left(\gamma^{-1}\right) T(\gamma) f(e)\right\| .
$$

These equations imply $(T(\gamma) f(e))^{*} \rho_{0}(\gamma)=\left(\rho_{0}\left(\gamma^{-1}\right) T(\gamma) f(e)\right)^{*}$ for every $\gamma \in S$. Since $\rho_{0}\left(\gamma^{-1}\right) o(\gamma)+o\left(\gamma^{-1}\right)=0$ holds, we obtain

$$
\begin{aligned}
\rho_{0}\left(\gamma^{-1}\right) T(\gamma) f(e) & =\rho_{0}\left(\gamma^{-1}\right) f(e)-\rho_{0}\left(\gamma^{-1}\right) \rho(\gamma) f(e) \\
& =\rho_{0}\left(\gamma^{-1}\right) f(e)-\rho_{0}\left(\gamma^{-1}\right) \rho_{0}(\gamma) f(e)-\rho_{0}\left(\gamma^{-1}\right) o(\gamma) \\
& =\rho_{0}\left(\gamma^{-1}\right) f(e)-f(e)+o\left(\gamma^{-1}\right) \\
& =-T\left(\gamma^{-1}\right) f(e) .
\end{aligned}
$$


Therefore we get $(T(\gamma) f(e))^{*} \rho_{0}(\gamma)=-\left(T\left(\gamma^{-1}\right) f(e)\right)^{*}$ for every $\gamma \in S$. Using $\left\|T\left(\gamma^{-1}\right) f(e)\right\|=\left\|\rho_{0}\left(\gamma^{-1}\right) T(\gamma) f(e)\right\|=\|T(\gamma) f(e)\|$, we have

$$
\begin{aligned}
& \sum_{\gamma \in S} m(\gamma)\|T(\gamma) f(e)\|\left(\operatorname{Re}(T(\gamma) f(e))^{*} \frac{v}{\|v\|}-\operatorname{Re}(T(\gamma) f(e))^{*} \rho_{0}(\gamma) \frac{v}{\|v\|}\right) \\
= & 2 \sum_{\gamma \in S} m(\gamma)\|T(\gamma) f(e)\| \operatorname{Re}(T(\gamma) f(e))^{*} \frac{v}{\|v\|} .
\end{aligned}
$$

Therefore we have

$$
\left|\nabla_{-} E\right|(f) \geq 2 \sum_{\gamma \in S} m(\gamma)\|T(\gamma) f(e)\| \operatorname{Re}(T(\gamma) f(e))^{*} \frac{v}{\|v\|} .
$$

On the other hand, the functions $\|T(\gamma) f(e)\|$ and $\operatorname{Re}(T(\gamma) f(e))^{*}$ are continuous on the space of $\rho$-equivariant maps. Hence the function

$$
\sum_{\gamma \in S} m(\gamma)\|T(\gamma) f(e)\| \operatorname{Re}(T(\gamma) f(e))^{*}
$$

is also continuous on the space of $\rho$-equivariant maps.

Next we review the definition of property $\left(E_{B}\right)^{\prime}$ and show Proposition 4.

Definition 7.4. We say that $\Gamma$ has property $\left(E_{B}\right)^{\prime}$ if, for any affine isometric $\Gamma$ representation $\rho$ whose linear part $\rho_{0}$ is nontrivial, there exists a positive constant $C(\rho)$ such that

$$
\left|\nabla_{-} E_{\rho^{\prime}}\right|(f)^{2} \geq C(\rho) E_{\rho^{\prime}}(f)
$$

for all $\rho^{\prime}$-equivariant maps $f$.

Note that a nontrivial affine isometric action $\rho$ whose linear part $\rho_{0}$ is trivial does not have a fixed point on $B$, but $E_{\rho^{\prime}}(f)>0=\left|\nabla_{-} E_{\rho^{\prime}}\right|(f)$ holds for all $\rho^{\prime}$-equivariant maps $f$. Hence, in Definition 7.4, we consider only affine isometric $\Gamma$-representations whose linear parts are nontrivial.

Proposition 4. Let $\Gamma$ be a finitely generated Abelian group, and $B$ a ucus Banach space. If $\Gamma$ has property $\left(T_{B}\right)$, then it has property $\left(E_{B}\right)^{\prime}$.

Proof. Take an arbitrary affine isometric $\Gamma$-representation $\rho$ on $B$ whose linear part $\rho_{0}$ is nontrivial. The quotient space $B^{\prime}$ is also a ucus Banach space; see [1]. By Proposition 7.3 , we have

$$
\left|\nabla_{-} E_{\rho^{\prime}}\right|(f) \geq \sum_{\gamma \in S} m(\gamma)\|T(\gamma) f(e)\| \frac{\operatorname{Re}(T(\gamma) f(e))^{*}\left(T_{0}(\gamma) v\right)}{\|v\|}
$$

for all $\rho^{\prime}$-equivariant maps $f: \Gamma \rightarrow B^{\prime}$ and nonzero vectors $v \in B^{\prime}$. Set $T(\gamma):=I d-$ $\rho^{\prime}(\gamma), T_{0}(\gamma):=I d-\rho_{0}^{\prime}(\gamma), O:=\sum_{\gamma \in S} m(\gamma)[o(\gamma)], X(f):=\sum_{\gamma \in S} m(\gamma) \rho_{0}^{\prime}(\gamma) f(e)$, $W(f):=X(f)+O$, and $V(f):=f(e)-W(f)=f(e)-X(f)-O$. 
In the case of $V(f) \neq 0$, we have

$$
\begin{aligned}
& \operatorname{Re}(T(\gamma) f(e))^{*}\left(T_{0}(\gamma) V(f)\right) \\
= & \operatorname{Re}(T(\gamma) f(e))^{*}\left(T_{0}(\gamma) f(e)-T_{0}(\gamma) W(f)\right) \\
= & \operatorname{Re}(T(\gamma) f(e))^{*}(T(\gamma) f(e)-T(\gamma) W(f)) \\
= & \|T(\gamma) f(e)\|-\operatorname{Re}(T(\gamma) f(e))^{*} T(\gamma) W(f) \\
\geq & \|T(\gamma) f(e)\|-\{\|T(\gamma) f(e)+T(\gamma) W(f)\|-\|T(\gamma) f(e)\|\} \\
= & 2\|T(\gamma) f(e)\|-\|T(\gamma) f(e)+T(\gamma) W(f)\|
\end{aligned}
$$

for every $\gamma \in S$. Because $\sum_{\kappa \in S} m(\kappa)=1$, we have

$$
\begin{aligned}
\|T(\gamma) f(e)+T(\gamma) W(f)\| & =\left\|\sum_{\kappa \in S} m(\kappa) T(\gamma) f(e)+T(\gamma)\left(\sum_{\kappa \in S} m(\kappa) \rho^{\prime}(\kappa) f(e)\right)\right\| \\
& =\left\|\sum_{\kappa \in S} m(\kappa)\left(T(\gamma) f(e)+T(\gamma) \rho^{\prime}(\kappa) f(e)\right)\right\|
\end{aligned}
$$

for every $\gamma \in S$. Since $\Gamma$ is Abelian, we have

$$
\begin{aligned}
T(\gamma) \rho^{\prime}(\kappa) f(e) & =\rho^{\prime}(\kappa) f(e)-\rho^{\prime}(\gamma) \rho^{\prime}(\kappa) f(e) \\
& =\rho^{\prime}(\kappa) f(e)-\rho^{\prime}(\kappa) \rho^{\prime}(\gamma) f(e) \\
& =\rho_{0}^{\prime}(\kappa) f(e)-\rho_{0}^{\prime}(\kappa) \rho^{\prime}(\gamma) f(e) \\
& =\rho_{0}^{\prime}(\kappa)(T(\gamma) f(e)) .
\end{aligned}
$$

Hence we get

$$
\begin{aligned}
\left\|\sum_{\kappa \in S} m(\kappa)\left(T(\gamma) f(e)+T(\gamma) \rho^{\prime}(\kappa) f(e)\right)\right\| & =\| \sum_{\kappa \in S} m(\kappa)\left(\left(I d+\rho_{0}^{\prime}(\kappa)\right)(T(\gamma) f(e)) \|\right. \\
& \leq \sum_{\kappa \in S} m(\kappa)\left\|\left(I d+\rho_{0}^{\prime}(\kappa)\right)(T(\gamma) f(e))\right\|
\end{aligned}
$$

for every $\gamma \in S$. By property $\left(T_{B}\right)$, for $\gamma \in S$ satisfying $T(\gamma) f(e) \neq 0$, there exists $\kappa_{\gamma} \in S$ such that

$$
\left\|\frac{T(\gamma) f(e)}{\|T(\gamma) f(e)\|}-\rho_{0}^{\prime}\left(\kappa_{\gamma}\right) \frac{T(\gamma) f(e)}{\|T(\gamma) f(e)\|}\right\| \geq C\left(\rho_{0}\right) .
$$

Since $B^{\prime}$ is uniformly convex, we have

$$
1-\frac{1}{2}\left\|\frac{T(\gamma) f(e)}{\|T(\gamma) f(e)\|}+\rho_{0}^{\prime}\left(\kappa_{\gamma}\right) \frac{T(\gamma) f(e)}{\|T(\gamma) f(e)\|}\right\| \geq \delta_{B^{\prime}}\left(C\left(\rho_{0}\right)\right) .
$$

Setting $\kappa_{\gamma}=\gamma$ for $\gamma \in S$ satisfying $\|T(\gamma) f(e)\|=0$, we have

$$
2\|T(\gamma) f(e)\|-\left\|T(\gamma) f(e)+\rho_{0}^{\prime}\left(\kappa_{\gamma}\right) T(\gamma) f(e)\right\| \geq 2 \delta_{B^{\prime}}\left(C\left(\rho_{0}\right)\right)\|T(\gamma) f(e)\|
$$

for every $\gamma \in S$. On the other hand, we have

$$
\|V(f)\| \leq \sum_{\gamma \in S} m(\gamma)\|T(\gamma) f(e)\| \leq\left(\sum_{\gamma \in S} m(\gamma)\|T(\gamma) f(e)\|^{2}\right)^{1 / 2} .
$$


Therefore we have

$$
\begin{aligned}
& \sum_{\gamma \in S} m(\gamma) \frac{\operatorname{Re}(T(\gamma) f(e))^{*}\left(T_{0}(\gamma) V(f)\right)}{\|V(f)\|}\|T(\gamma) f(e)\| \\
\geq & \sum_{\gamma \in S} m(\gamma) \frac{\sum_{\kappa \in S} m(\kappa)\left(2\|T(\gamma) f(e)\|-\left\|\left(I d+\rho_{0}^{\prime}(\kappa)\right)(T(\gamma) f(e))\right\|\right)}{\|V(f)\|}\|T(\gamma) f(e)\| \\
\geq & \sum_{\gamma \in S} m(\gamma) \frac{m\left(\kappa_{\gamma}\right)\left(2\|T(\gamma) f(e)\|-\left\|\left(I d+\rho_{0}^{\prime}\left(\kappa_{\gamma}\right)\right)(T(\gamma) f(e))\right\|\right)}{\|V(f)\|}\|T(\gamma) f(e)\| \\
\geq & \sum_{\gamma \in S} m(\gamma) \frac{\min _{\kappa \in S} m(\kappa) 2 \delta_{B^{\prime}}\left(C\left(\rho_{0}\right)\right)\|T(\gamma) f(e)\|}{\left(\sum_{\lambda \in S} m(\lambda)\|T(\lambda) f(e)\|^{2}\right)^{1 / 2}}\|T(\gamma) f(e)\| \\
= & \min _{\kappa \in S} m(\kappa) 2 \delta_{B^{\prime}}\left(C\left(\rho_{0}\right)\right)\left(\sum_{\gamma \in S} m(\gamma)\|T(\gamma) f(e)\|^{2}\right)^{1 / 2} \\
= & \min _{\kappa \in S} m(\kappa) 2 \sqrt{2} \delta_{B^{\prime}}\left(C\left(\rho_{0}\right)\right) E_{\rho^{\prime}}(f)^{1 / 2}
\end{aligned}
$$

that is,

$$
\left|\nabla_{-} E_{\rho^{\prime}}\right|(f)^{2} \geq 8 \delta_{B^{\prime}}\left(C\left(\rho_{0}\right)\right)^{2} \min _{\kappa \in S} m(\kappa)^{2} E_{\rho^{\prime}}(f)
$$

Set $C(\rho):=8 \delta_{B^{\prime}}\left(C\left(\rho_{0}\right)\right)^{2} \min _{\kappa \in S} m(\kappa)^{2}$.

In the case of $V(f)=0$ and $O \neq 0$, since $f(e)-X(f)=O$ we have

$$
V(a f)=a f(e)-X(a f)-O=a(f(e)-X(f))-O=(a-1) O \neq 0
$$

for $a \neq 1$. Thus, using the result in the case of $V(f) \neq 0$ and Proposition 7.3 , we have

$$
\begin{aligned}
& \lim _{a \nearrow 1} C(\rho)^{1 / 2} E_{\rho^{\prime}}(a f)^{1 / 2} \\
\leq & \lim _{a \nearrow 1} \sum_{\gamma \in S} m(\gamma) \frac{\operatorname{Re}(T(\gamma)(a f(e)))^{*}\left(T_{0}(\gamma) V(a f)\right)}{\|V(a f)\|}\|T(\gamma)(a f(e))\| \\
= & \lim _{a \nearrow 1} 2 \sum_{\gamma \in S} m(\gamma)\|T(\gamma)(a f(e))\| \operatorname{Re}(T(\gamma)(a f(e)))^{*} \frac{V(a f)}{\|V(a f)\|} \\
= & 2 \sum_{\gamma \in S} m(\gamma)\|T(\gamma) f(e)\| \operatorname{Re}(T(\gamma) f(e))^{*} \frac{-O}{\|O\|} \\
\leq & \left|\nabla_{-} E_{\rho^{\prime}}\right|(f) .
\end{aligned}
$$

Since $E_{\rho^{\prime}}$ is continuous, we have $\left|\nabla_{-} E_{\rho^{\prime}}\right|(f)^{2} \geq C(\rho) E_{\rho^{\prime}}(f)$.

In the case of $V(f)=0$ and $O=0$, we suppose $f(e) \neq 0$. By property $\left(T_{B}\right)$, there exists $\gamma_{1} \in S$ such that $\left\|\rho_{0}^{\prime}\left(\gamma_{1}\right) f(e)-f(e)\right\| \geq C\left(\rho_{0}\right)\|f(e)\|$. Since $B^{\prime}$ is uniformly convex, we have

$$
1-\frac{\left\|\rho_{0}^{\prime}\left(\gamma_{1}\right) f(e)+f(e)\right\|}{2\|f(e)\|}>\delta_{B^{\prime}}\left(C\left(\rho_{0}\right)\right) .
$$


Since $V(f)=0$ and $O=0$, we have $f(e)=X(f)$. Hence we have

$$
\begin{aligned}
\|2 f(e)\| & =\|f(e)+X(f)\| \\
& =\left\|\sum_{\gamma \in S} m(\gamma)\left(f(e)+\rho_{0}^{\prime}(\gamma) f(e)\right)\right\| \\
& \leq \sum_{\gamma \in S} m(\gamma)\left\|f(e)+\rho_{0}^{\prime}(\gamma) f(e)\right\| \\
& <m\left(\gamma_{1}\right)\left(2-2 \delta_{B^{\prime}}(\epsilon)\right)\|f(e)\|+\sum_{\gamma \in S, \gamma \neq \gamma_{1}} m(\gamma)\left\|f(e)+\rho_{0}^{\prime}(\gamma) f(e)\right\| \\
& \leq 2\|f(e)\|-2 \delta_{B^{\prime}}(\epsilon) m\left(\gamma_{1}\right)\|f(e)\| \\
& <\|2 f(e)\| .
\end{aligned}
$$

This is a contradiction. Hence $f(e)=0$, and a $\rho^{\prime}$-equivariant map $g$ with $g(e) \neq 0$ satisfies $V(a g) \neq 0$ for $0<a<1$. Since $V(a g)=a(g(e)-X(g))=a V(g)$, as in the case of $V(f)=0$ and $O \neq 0$, we have

$$
\begin{aligned}
& \lim _{a \searrow 0} C(\rho)^{1 / 2} E_{\rho^{\prime}}(a g)^{1 / 2} \\
\leq & \lim _{a \searrow 0} \sum_{\gamma \in S} m(\gamma)\|T(\gamma)(a g(e))\| \frac{\operatorname{Re}(T(\gamma)(a g(e)))^{*}\left(T_{0}(\gamma) V(a g)\right)}{\|V(a g)\|} \\
= & \lim _{a \searrow 0} 2 \sum_{\gamma \in S} m(\gamma)\|T(\gamma)(a g(e))\| \operatorname{Re}(T(\gamma)(a g(e)))^{*} \frac{V(a g)}{\|V(a g)\|} \\
= & 2 \sum_{\gamma \in S} m(\gamma)\|T(\gamma) f(e)\| \operatorname{Re}(T(\gamma) f(e))^{*} \frac{V(g)}{\|V(g)\|} \\
\leq & \left|\nabla_{-} E_{\rho^{\prime}}\right|(f) .
\end{aligned}
$$

Therefore we have $\left|\nabla_{-} E_{\rho^{\prime}}\right|(f)^{2} \geq C(\rho) E_{\rho^{\prime}}(f)$.

Note that the assumption that $\Gamma$ is Abelian is only used in the case of $V(f) \neq 0$. Hence, if there exists a positive constant $C$ satisfying $\left|\nabla_{-} E_{\rho^{\prime}}\right|(f)^{2} \geq C E_{\rho^{\prime}}(f)$ for all $f \in \mathcal{M}$ with $V(f) \neq 0$, we can show Proposition 4 without the assumption.

\section{REFERENCES}

1. U. Bader, A. Furman, T. Gelander, and N. Monod, Property $(T)$ and rigidity for actions on Banach spaces, Acta Math. 198 (2007), no.1, 57-105. MR2316269 (2008g:22007)

2. M. R. Bridson and A. Haefliger, Metric spaces of non-positive curvature, Grundlehren der Mathematischen Wissenschaften [Fundamental Principles of Mathematical Sciences], 319. Springer-Verlag, Berlin, 1999. MR,1744486 (2000k:53038)

3. Y. Benyamini and J. Lindestrauss, Geometric nonlinear functional analysis. Vol. 1. American Mathematical Society Colloquium Publications, 48. American Mathematical Society, Providence, RI, 2000. MR:1727673 (2001b:46001)

4. H. Busemann, The geometry of geodesics, Academic Press Inc., New York, N. Y., 1955. MR:0075623 (17:779a)

5. A. Guichardet, Sur la cohomologie des groupes topologiques. II, Bull. Sci. Math. (2) 96 (1972), 305-332. MR0340464 (49:5219)

6. H. Izeki, T. Kondo and S. Nayatani, Fixed-point property of random groups, Ann. Global Anal. Geom. 35 (2009), no. 4, 363-379. MR2506240

7. H. Izeki and S. Nayatani, Combinatorial harmonic maps and discrete-group actions on Hadamard spaces, Geom. Dedicata 114 (2005), 147-188. MR2174098(2006k:58024)

8. J. Jost, Nonpositive curvature: geometric and analytic aspects, Lectures in Math. ETH Zürich. Birkhäuser Verlag, Basel, 1997. MR1451625 (98g:53070) 
THE ENERGY OF EQUIVARIANT MAPS AND A FIXED-POINT PROPERTY

9. J. Jost, Nonlinear Dirichlet forms, New directions in Dirichlet forms, 1-47, AMS/IP Stud. Adv. Math., 8, Amer. Math. Soc., Providence, RI, 1998. MR.1652278 (99k:31012)

10. M. Kapovich, Hyperbolic manifolds and discrete groups, Progress in Mathematics, 183. Birkhäuser Boston, Inc., Boston, MA, 2001. MR.1792613 (2002m:57018)

11. U. F. Mayer, Gradient flows on nonpositively curved metric spaces and harmonic maps, Comm. Anal. Geom. 6 (1998), 199-253. MR1651416 (99m:58067)

12. S. Ohta, Convexities of metric spaces, Geom. Dedicata 125 (2007), 225-250. MR2322550 (2008j:53063)

13. A. Papadopoulos, Metric spaces, convexity and nonpositive curvature, IRMA Lectures in Mathematics and Theoretical Physics, 6. European Mathematical Society (EMS), Zürich, 2005. MR2132506 (2005k:53042)

Mathematical Institute, Tohoku University, Sendai 980-8578, Japan

E-mail address: sa5m10@math.tohoku.ac.jp 